\title{
Solution-processed electrical doping of organic semiconductors and their application for organic devices
}

\begin{abstract}
An economically expedient and simple method to improve charge injection and transport through organic semiconductors via solution-processed low-concentration doping is suggested. Two organic species, Pyronin B (PyB) and Acridine Orange (AO) dissolved in 2-butanone, have been used as dopants for a conjugated polymer. Ultraviolet photoelectron spectroscopy (UPS) and UV-vis-NIR absorption spectroscopy studies confirmed that charge transfer between the organic semiconductor and dopants took place successfully. It should also be noted that the doping efficiency of the dopants is strongly dependent on the intrinsic properties of the conjugated polymers.
\end{abstract}

Keywords: organic devices, solution-processed doping, organic semiconductors, organic dopants, charge transfer, conjugated polymers
Volume 3 Issue 3 - 2019

\section{Gunel Huseynova}

Dongguk University, South Korea

Correspondence: Huseynova G, Dongguk University, Pildongro 30, Jung-gu, Seoul, South Korea, Tel +82 I0 3064-5407, Email bblg7839@gmail.com

Received: March 28, 2019 | Published: June 28, 2019

\section{Introduction}

Different electronic devices based on organic semiconductors (OSCs) are being developed and promoted every year due to unique and outstanding properties of the organic materials including flexibility, transparency, light weight, and solution-process ability. However, these materials are not fully commercialized due to their intrinsically low electrical performance and poor stability. And in order to overcome these issues several approaches have been developed and one of them is doping. Doping is the most straightforward method to increase electrical conductivity of the materials in the first place. The organic light-emitting diode (OLED) industry already uses this method to finally commercialize these organic devices successfully. ${ }^{1}$ It should be noted that doping of OSCs is completely different from that of inorganic ones in which conductivity is enhanced via the increase of charge carriers provided by the impurity atoms that replace the atoms in the host lattice. In organic electronics, no replacement of host lattice atoms by impurity atoms occurs. Rather, it is just simple and direct introduction of extra charge carriers to the whole host molecule via a charge transfer process. ${ }^{2}$ In this case, the effectiveness of the doping process depends on the energy level differences between the materials' highest occupied molecular orbital (HOMO) and lowest unoccupied molecular orbital (LUMO). This requirement seriously limits the doping possibilities of the organic materials as dopant and host molecules with matching energy levels are rare. One of the suggested alternatives for doping of OSCs in which the energy levels of the two materials can be ignored, is application of Lewis acids and bases for $\mathrm{p}$ - and n-type doping, respectively. ${ }^{3-7}$ In this work, two cationic organic dyes, Pyronin B (PyB) and Acridine Orange (AO), are investigated as p-type dopants for a conjugated ambipolar polymer diketopyrrolopyrrole-thieno [3,2-b]thiophene (DPPT-TT). The dopants are conjugated molecules with Lewis acid nature.

\section{Experimental}

The polymer solution were prepared in 1,2-dichlorobenzene (DCB) at the concentration of $10 \mathrm{mg} / \mathrm{ml}$. Small amounts $(0.5,2$, and $10 \mathrm{mg}$ ) of the dopants were dissolved in different organic solvents, such as ethanol, 2-propanol, isopropyl alcohol, ethyl acetate, and a mixture of organic solvents (consisting of toluene $(50 \%)$, ethanol $(15 \%)$, butyl-acetate $(10 \%)$, butanol $(10 \%)$, 2-ethoxyethanol $(8 \%)$, and acetone (7\%)) to form solutions of $0.5 \mathrm{mg} / \mathrm{ml}$ in ethanol; $2 \mathrm{mg} / \mathrm{ml}$ in 2-propanol, isopropyl alcohol, and ethyl acetate; $10 \mathrm{mg} / \mathrm{ml}$ in the above mentioned solvent mixture (Figure 1).
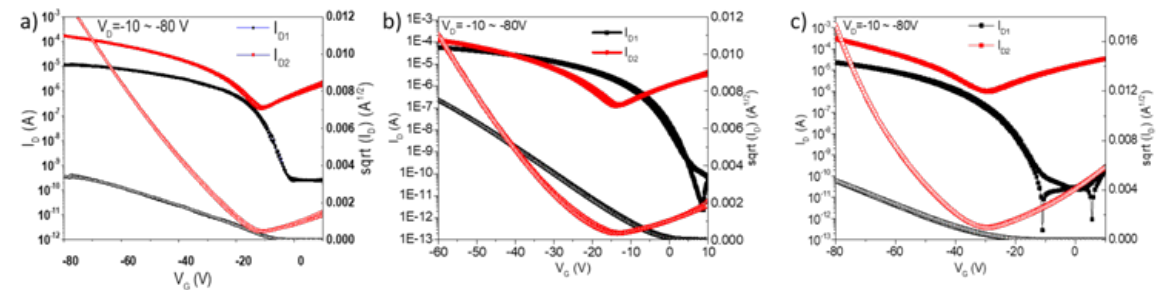

Figure I The OFET transfer characteristics of the doped: A) PyB-doped and B) AO-doped and (C) Illustrates the intrinsic OFET performance of DPPT-TT polymer. As seen, DPPT-TT exhibits almost ideal ambipolar performance. But after doping with the cationic dyes, the ambipolar device performance changed to unipolar, and the on/off current ratio was also improved by doping. The undoped and doped devices exhibit on/off ratios of $10^{2}$, I $0^{3}$ (for $A O-$ doped devices), and $10^{4}$ (for PyB-doped devices, respectively. 


\section{Results and discussion}

Dopants were dissolved in 2-butanone and applied by mixing them in a single blend system with the host in the same solution (film doping). Dopant solutions were added into the host solution of DPPTTT at different volume concentrations and mixed with magnetic stirrer on a hot plate for more than 24hours for complete blending. By optimization of the dopant concentration, the whole transport of the material was enhanced. The doped polymer films were applied to organic field-effect transistors (OFETs). As it is clear from Figure 2A, for doped DPPT-TT films, the Fermi level (EF) gradually shifted towards the DPPT-TT HOMO, which clearly indicates increased hole density by PyB doping. The HOMO and LUMO levels of the pristine DPPT-TT were 5.33 and $4.07 \mathrm{eV}$, respectively. According to previous studies, PyB has a reduction potential similar to that of Pyronin Y, which is $-0.445 \mathrm{~V}$ versus the normal hydrogen electrode (NHE) ${ }^{1,3,4}$ And the LUMO level of Pyronin $\mathrm{Y}$ is $-4.685 \mathrm{eV}$, then, which is calculated from its reduction potential following the conventional formula of $\mathrm{ELUMO}=-($ Ered +5.13$) \mathrm{eV}(5.13 \mathrm{eV}$ is the constant (the absolute electrode potential) value representing the potential difference between the energy level of the NHE and the vacuum level). So, PyB also has a LUMO level around $\sim-4.685 \mathrm{eV}$. Considering the energy levels and electron accepting property of $\mathrm{PyB}$, efficient electron transfer is expected from DPPT-TT to PyB for p-type doping. This type of doping is achieved using a strong $\pi$-electron accepting Lewis acid species. The charge transfer process between the polymer and $\mathrm{PyB}$ is assumed to be as follows:

$$
\text { DPPT-TT0 }+ \text { PyB }+=\text { DPPT-TT }++ \text { PyB } 0
$$

Through the generation of free charge carriers by doping, the Fermi level shifted towards HOMO by $0.19,0.23$, and $0.3 \mathrm{eV}$ for 1:40, 1:10, and 1:7 doping ratio, respectively. The Fermi level shift and optical band gap values were calculated from UPS and UV-vis absorption spectra respectively, and are given in Figure 2B \& Table 1.
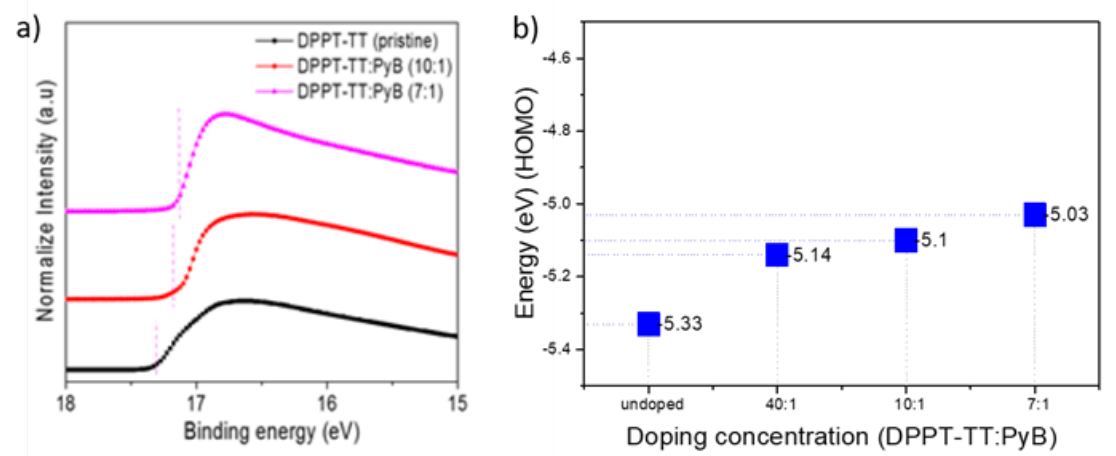

Figure 2 A) UPS spectra of pristine and doped DPPT-TT films; B) Band gap and HOMO level values of the pristine and doped DPPT-TT films.

Table I Extracted Highest Occupied Molecular Orbital (HOMO) level and band gap values

\begin{tabular}{lll}
\hline $\begin{array}{l}\text { Doping concentration (DPPT- } \\
\text { TT:PyB, weight ratio) }\end{array}$ & HOMO $(\mathrm{eV})$ & Optical band gap $(\mathrm{eV})$ \\
\hline pristine & -5.33 & 1.26 \\
40:01:00 & -5.14 & 1.07 \\
10:01 & -5.1 & 1.03 \\
7:01 & -5.03 & 0.96 \\
\hline
\end{tabular}

\section{Conclusion}

Two Lewis acids, PyB and AO were studied as p-type dopants for a conjugated polymer DPPT-TT. The doped devices showed highly improved device characteristics with enhanced hole transport. Although these dopants are not stable in air, this simple solution doping can be applied as an effective alternative method to enhance the electrical performance of p-type conjugated polymers.

\section{Acknowledgments}

This work was supported by the National Research Foundation of Korea (NRF) grant funded by the Korean Government (MSIP)
(NRF-2014R1A2A2A01007159), by the Center for Advanced Soft-Electronics (2013M3A6A5073183) funded by the Ministry of Science, ICT and Future Planning.

\section{Conflicts of interest}

Authors declare that there is no conflict of interest.

\section{References}

1. Salzmann I, Heimel G. Toward a comprehensive understanding of molecular doping organic semiconductors (review). Journal of Electron Spectroscopy and Related Phenomena. 2015;204:208-222. 
2. Lüssem B, Riede M, Leo K. Doping of Organic Semiconductors. Phys Status Solidi A. 2013;210(1):9-43.

3. Panidi J, Paterson FA, Khim D, et al. Remarkable Enhancement of the Hole Mobility in Several Organic Small-Moelcules, Polymers, and SmallMolecule:Polymer Blend Transistors by Simple Admixing of the Lewis Acid P-Dopant $\mathrm{B}\left(\mathrm{C}_{6} \mathrm{~F}_{5}\right)_{3}$. Adv Sci. 2018;5: 1700290.

4. Pingel P, Arvind M, Kölln 1, et al. p-type Doping of Poly(3-Hexylthiophene) with the Strong Lewis Acid Tris(pentafluorophenyl)borane. Adv Electron Mater. 2016;2:1600204

5. Xu Y, Sun H, Shin EY, et al. Planar-Processed Polymer Transistors. Adv Mater. 2016;28:8531-8537.
6. Li H, Risko C, Seo HJ, et al. Fullerene-Carbene Lewis Acid-Base Adducts. $J$ Am Chem Soc. 2011;133:12410-12413.

7. Huseynova G, Xu Y, Yawson BN, et al. P-Type Doped Ambipolar Polymer Transistors by Direct Charge Transfer from a Cationic Dye Pyronin B Ferric Chloride. Org Electron. 2016;39:229-235. 